\title{
Evaluation on prebiotic properties of $\beta$-glucan and oligo- $\beta$-glucan from mushrooms by human fecal microbiota in fecal batch culture
}

\section{Chiraphon Chaikliang ${ }^{1}$,Santad Wichienchot ${ }^{1}$, Wirote Youravoug $^{2}$ and Potchanapond Graidist $^{3}$}

${ }^{1}$ Interdisciplinary Graduate School of Nutraceutical and Functional Food, Songkla University, Hat Yai, Songkhla 90112, Thailand; ${ }^{2}$ Department of Food Technology, Faculty of AgroIndustry, Songkla University, Hat Yai, Songkhla 90112, Thailand; ${ }^{3}$ Department of Biomedical Science, Faculty of Medicine Prince of Songkla University, Hat Yai, Songkhla 90112, Thailand

Corresponding Author: Chiraphon Chaikliang, Interdisciplinary Graduate School of Nutraceutical and Functional Food, Songkla University, Hat Yai, Songkhla 90112, Thailand

Submission Date: July 17, 2015, Acceptance date: November 25, 2015: Publication date: November 29, 2015

\begin{abstract}
:
Background: $\beta$-glucan is dietary fiber, a structural polysaccharide, $\beta$-linked linear chains of Dglucose polymers with variable frequency of branches. $\beta$-glucan is isolated from different sources such as cell walls of baker's yeast (Saccharomyces cerevisiae), cereals (oat and barley) and various species of mushrooms. Among 8 mushrooms in the study, Schizophylum commune Fr and Auricularia auricula Judae had the highest in $\beta$-glucan contents and the cheapest cost of mushroom per content of $\beta$-glucan, respectively. Even the function of $\beta$-glucan on immune modulation has been known however no report on interaction between $\beta$-glucan and human gut microbiota. Gut microbiota is thought to have health effects by interaction with non-digestible component particular fermentable dietary fiber. It is important to correlate the specific groups of the microbial communities associated with $\beta$-glucan fermentation and the consequential SCFA profiles. $\beta$-glucan from mushroom may has potential prebiotic function similar to those from commercial yeast (Saccharomyces cerevisiae) $\beta$-glucan.
\end{abstract}

Objective: To evaluate on prebiotic properties of soluble $\beta$-glucans and oligo- $\beta$-glucans from Schizophylum commune Fr and Auricularia auricula Judae by fecal fermentation in batch culture.

Methods: In vitro fecal fermentation in anaerobic batch cultures under simulated conditions similar to human colon with human faecal samples from three donors were performed. Comparison on $3 \beta$-glucans and 2 oligo- $\beta$-glucans have been studied. Sample was taken at $0 \mathrm{~h}$, $24 \mathrm{~h}$ and $48 \mathrm{~h}$ to analyze the numbers of bacterial changes by fluorescent in situ hybridization (FISH) technique. Short chain fatty acids (SCFA) were analyzed by HPLC. The prebiotic index 
(PI) was calculated according to the change of 5 specific bacterial genus within $48 \mathrm{~h}$ fermentation.

Results: Soluble $\beta$-glucan from Auricularia auricula Judae increased numbers of bifidobacteria and lactobacillus significantly $(\mathrm{P}<0.05)$. The PI of soluble $\beta$-glucan and oligo- $\beta$-glucan from Schizophylum commune Fr were 0.01 and -0.01 , respectively. $\beta$-glucan and oligo- $\beta$-glucan from Auricularia auricula Judae were 0.11 and -0.07 , respectively. Whereas PI of $\beta$-glucan from commercial yeast (Saccharomyces cerevisiae) was 0.03 . Acetate was the most prevalent SCFA found in all treatments followed by propionate, butyrate and lactate, respectively.

Conclusion: The study confirmed that $\beta$-glucan from Schizophylum commune Fr and Auricularia auricula Judae are candidate prebiotics.

Keywords: $\beta$-glucan, oligo- $\beta$-glucan, prebiotic, mushroom, fecal batch culture

\section{INTRODUCTION:}

Prebiotics are non-digested food ingredients that beneficially affects the host by selectively stimulating the growth and/or activity of one or a limited number of bacteria in the colon", that can improve the host health [1]. The usual target species for such a dietary intervention are bifidobacteria and lactobacillus. These organisms have been reported to have health-promoting properties such as inhibition of exogenous pathogens. Many studies have now confirmed that prebiotics are a valid approach to the dietary manipulation of the colonic microflora [2]. In addition to the desirable effect of increased bifidobacteria and lactobacillus while suppressing, or not affecting, less desirable bacteria, such as proteolytic bacteroides and clostridia [3], shortchain fatty acids (SCFA) are produced as the end products of $\beta$-glucans fermentation [2].

Among the emerging prebiotic candidates, $\beta$-glucans are receiving increasing attention due to their different origins and structural diversity as well as fermentation characteristics and human health benefits $[4,5]$. $\beta$-glucan is dietary fiber, a structural polysaccharide, $\beta$-linked linear chains of D-glucose polymers with variable frequency of branches. $\beta$-Glucan isolated from different sources such as cell walls of baker's yeast (Saccharomyces cerevisiae), cereals (oat and barley) and various species of mushrooms. In their natural states, yeast and mushroom contains a mixture of $\beta$-1,3-glucans and $\beta$-1,6-glucans. oat and barley contains a mixture of $\beta$-1,3-glucans and $\beta$-1,4-glucans [6]. It was reported that laminarin, a linear $\beta$-1,3-glucan isolated from seaweed Laminaria digitata, could be highly fermented by human fecal inoculum in $24 \mathrm{~h}$, and a total short-chain fatty acid (SCFA) concentration of $85 \mathrm{mM}$ was produced with $60 \%$ acetic acid. In another study, when curdlan, a $\beta$-1,3-glucan from the bacteria Alcaligenes faecalis, was fed to rats for 4 weeks, there was a significant increase in bifidobacterial proliferation as well as production of SCFAs and lactate in the animal's fecal contents. $\beta$-Glucan in cereals contains mixed $\beta$-1,3- and $\beta$-1,4-glycosidic linkages. Fermentation of $\beta$-glucans from barley and oat by the human faecal microbiota gave a unique SCFA profile that is high in propionic acid which might exert a hypocholesterolaemic effect. $\beta$-Glucan from the mushroom sclerotia of Pleurotus tuber-regium, which is a highly branched $\beta$-1,3-linked polysaccharide, was reported to have antitumor and immunomodulating activities. In an in vitro study, $\beta$-glucans from three 
mushroom sclerotia were fermented by human fecal homogenate to different extents due to their structural differences. Mushroom $\beta$-glucans from Poria cocos and Polyporous rhinocerus stimulated growth of Lactobacillus brevis and Bifidobacterium longum but inhibited proliferation of Clostridium celatum in a $24 \mathrm{~h}$ in vitro fermentation study [7]. Furthermore, many research studies have indicated that polysaccharides obtained from mushrooms such as Pleurotus sp., Lentinus edodes, Tremella fuciformis and Agaricus bisporus have prebiotic activity. The active component is believed to be the long chain beta-glucans, including homo-glucans and heteroglucans with $\beta(1 \rightarrow 3), \beta(1 \rightarrow 4)$, and $\beta(1 \rightarrow 6)$ glucosidic linkages [8].

This investigation was evaluated the fermentation properties of soluble $\beta$-glucans and oligo$\beta$-glucans from 2 types of mushrooms and commercial yeast $\beta$-glucan. Method for production of oligo- $\beta$-glucans were succeeded by hydrolysis of mushroom $\beta$-glucans with $0.00145 \mathrm{U} / \mathrm{ml}$ laminarinase (MP Biomedicals, USA), pH 5 at $37{ }^{\circ} \mathrm{C}$ for 9 and $12 \mathrm{~h}$. Fecal batch fermentation was performed under anaerobic conditions mimic colon fermentation. Culture $\mathrm{pH}$ was controlled at 6.8 and stirred throughout experiment for $48 \mathrm{~h}$. Assumption of the study is $\beta$-glucan from different sources and molecular weight distributions may influence on the fermentation patterns by human faecal microbiota.

\section{MATERIALS AND METHODS:}

\section{Materials}

Soluble $\beta$-glucans from Schizophylum commune $\mathrm{Fr}$ and Auricularia auricula Judae were prepared by extraction with water under high pressure $(270 \mathrm{kPa})$ and high temperature $\left(130{ }^{\circ} \mathrm{C}\right)$ for 30 and $90 \mathrm{~min}$. The extract was spray dried by a mini spray dryer (B-290, Buchi 290, Switzerland) at inlet temperature of $180{ }^{\circ} \mathrm{C}$ to obtain $\beta$-glucan powders.

Soluble oligo- $\beta$-glucans were prepared by hydrolysis of $\beta$-glucans from Schizophylum commune Fr and Auricularia auricula Judae with $00145 \mathrm{U} / \mathrm{ml}$ laminarinase, $37{ }^{\circ} \mathrm{C}$, for 9 and 12 $\mathrm{h}$. The extract was spray dried to obtain oligo- $\beta$-glucan powders by a mini spray dryer.

Commercial yeast (Saccharomyces cerevisiae) $\beta$-glucan was purchased from Y\&L Biotech Co., Ltd, China. All chemicals were purchased from Sigma, Poole, UK, otherwise it was specified.

\section{Methods}

\section{In vitro fecal fermentation in batch culture}

Batch cultures under anaerobic fecal fermentation were used to study the growth of fecal bacteria during fermentation of the soluble $\beta$-glucans and oligo- $\beta$-glucans from mushrooms and commercial yeast $\beta$-glucan. Batch culture system consisted of a water-jacket glass vessel (320 $\mathrm{ml}$ ), maintained at $37^{\circ} \mathrm{C}$, that was filled with pre-reduced basal culture medium, and the $\mathrm{pH}$ maintained by $\mathrm{pH}$-controller at $6.8 \pm 0.2$ throughout the experiment. The basal medium contained (per litre): 2 g peptone water, $2 \mathrm{~g}$ yeast extract, $0.1 \mathrm{~g} \mathrm{NaCl}, 0.04 \mathrm{~g} \mathrm{~K}_{2} \mathrm{HPO}_{4}, 0.04 \mathrm{~g} \mathrm{KH}_{2} \mathrm{PO}_{4}, 0.01$ $\mathrm{g} \mathrm{MgSO}_{4} .7 \mathrm{H}_{2} \mathrm{O}, 0.01 \mathrm{~g} \mathrm{CaCl}_{2} \cdot 6 \mathrm{H}_{2} \mathrm{O}, 2 \mathrm{~g} \mathrm{NaHCO}_{3}, 0.005 \mathrm{~g}$ haemin, $0.5 \mathrm{~g}$ L-cysteine $\mathrm{HCl}, 0.5 \mathrm{~g}$ bile salts, $2 \mathrm{ml}$ Tween $80,10 \mathrm{ml}$ vitamin $\mathrm{K}$, and $4 \mathrm{ml}$ of $0.025 \%(\mathrm{w} / \mathrm{v})$ resazurin solution. The medium was adjusted to $\mathrm{pH} 7.0 \mathrm{using} 1 \mathrm{~mol} \mathrm{l}^{-1} \mathrm{HCl}$. The medium were placed in an anaerobic cabinet at $37^{\circ} \mathrm{C}$ overnight to pre-reduce the media. A $10 \%(\mathrm{w} / \mathrm{v})$ faecal slurry was prepared using fresh faeces from three donors (who had not taken antibiotics for 3 months beforehand) and pre- 
reduced $\left(0.1 \mathrm{~mol} \mathrm{l}^{-1}\right)$ phosphate buffer $(\mathrm{pH} 7.0)$ was mixed in a stomacher for $2 \mathrm{~min}$. The soluble $\beta$-glucans and oligo- $\beta$-glucans were added to give a final concentration of $1 \%(\mathrm{w} / \mathrm{v})$. Each vessel was magnetically stirred and the temperature set at $37^{\circ} \mathrm{C}$ by a circulating water bath. Culture $\mathrm{pH}$ was controlled automatically and maintained at $\mathrm{pH} 6.8 \pm 0.2$. Anaerobic conditions were maintained by sparging the vessels with oxygen-free nitrogen gas. Samples $(5 \mathrm{ml})$ were taken from each vessel at start $\left(\mathrm{T}_{0}\right), 24 \mathrm{~h}$ and $48 \mathrm{~h}$ incubation period for enumeration of bacteria by fluorescent in situ hybridization (FISH) technique and short chain fatty acids (SCFA) were analyzed by HPLC [2].

\section{Enumeration of fecal bacteria}

Static batch culture fermentations were set up as described above and samples were removed for bacterial enumeration by fluorescent in situ hybridization (FISH) technique. At 0,24 and $48 \mathrm{~h}$ fermentation, samples $(375 \mu \mathrm{l})$ were removed from the batch cultures and added to $1,125 \mu \mathrm{l}$ filtered $4 \%(\mathrm{w} / \mathrm{v})$ paraformaldehyde solution $(\mathrm{pH} 7.2)$, mixed and stored at $4{ }^{\circ} \mathrm{C}$ overnight to fix the cells. The fixed cells were washed twice in filtered $0.1 \mathrm{~mol} \mathrm{l}^{-1}$ phosphate buffer solution $(\mathrm{pH}$ 7.0) and resuspended in $150 \mu \mathrm{l}$ phosphate buffer solution (0.1 $\left.0.1 \mathrm{~mol} \mathrm{l^{-1 }}, \mathrm{pH} 7.0\right)$. Ethanol 96\% $(150 \mu \mathrm{l})$ was added and the sample mixed and stored at $20^{\circ} \mathrm{C}$ until needed, but no longer than 3 months. Hybridization was carried out at the appropriate temperature using genus-specific $16 \mathrm{~S}$ rRNA-targeted oligonucleotide probes labeled with the fluorescent dye Cy3 for the different bacterial groups or with 4, 6-diamidino-2-phenylindole for total cell counts. The bacterial groups were selected based on their high abundance within, and contribution to, the colonic microbiota. The probes used were Bif 164 specific for Bifidobacterium $\left(50^{\circ} \mathrm{C}\right)$, Bac 303 specific for Bacteroides $\left(48^{\circ} \mathrm{C}\right)$, Lab 158 specific for Lactobacillus/ Enterococcus spp. $\left(50^{\circ} \mathrm{C}\right)$, His 150 specific for Clostridium $\left(50^{\circ} \mathrm{C}\right)$, Eub 338 specific for Eubacterium $\left(48^{\circ} \mathrm{C}\right)$. Cells were counted using a fluorescent microscope (Nikon, Japan). At least 15 random fields, each containing $30 \pm 300$ cells, were counted for each slide [2].

\section{Prebiotic index (PI)}

To obtain a general quantitative measure of the prebiotic effect, a prebiotic index (PI) was calculated for the soluble $\beta$-glucans and oligo- $\beta$-glucans. The PI equation was described as follows [9]:

$$
\begin{gathered}
\text { Prebiotic index }(\mathrm{PI})=\alpha+\beta-\gamma-\delta \\
\alpha=\left(\mathrm{Bif}_{\mathrm{t}} / \mathrm{Bif}_{0}\right) / \text { Total } \\
\beta=\left(\mathrm{Lac}_{\mathrm{t}} / \mathrm{Lac}_{0}\right) / \text { Total } \\
\gamma=\left(\mathrm{Bac}_{\mathrm{t}} / \mathrm{Bac}_{0}\right) / \text { Total } \\
\delta=\left(\mathrm{Clos}_{\mathrm{t}} / \mathrm{Clos}_{0}\right) / \text { Total }
\end{gathered}
$$

Bif $_{\mathrm{t}}=$ Bifidobacteria count at $\mathrm{t}=24$ or 48 hours $/$ Bif $_{0}=$ Bifidobacteria count 0 hour

$\mathrm{Lac}_{\mathrm{t}}=$ Lactobacilli count at $\mathrm{t}=24$ or 48 hours $/ \mathrm{Lac}_{0}=$ Lactobacilli count 0 hour

$\mathrm{Bac}_{\mathrm{t}}=$ Bacteroides count at $\mathrm{t}=24$ or 48 hours $/ \mathrm{Bac}_{0}=$ Bacteroides count 0 hour

Clos $_{\mathrm{t}}=$ Clostridia count at $\mathrm{t}=24$ or 48 hours $/ \mathrm{Clos}_{0}=\mathrm{Clostridia}$ count 0 hour

Total $=\operatorname{Eub}_{\mathrm{t}} / \mathrm{Eub}_{0}($ Eubacteria $)$ total count at $\mathrm{t}=24$ or 48 hours $/$ total count at 0 hour 


\section{Short chain fatty acid analysis}

Samples taken from the vessels were centrifuged $\left(13,000 \mathrm{xg}\right.$ for $\left.15 \min 4{ }^{\circ} \mathrm{C}\right)$, and the supernatant was passed through a $0.2 \mu \mathrm{m}$ filter before short chain fatty acid (SCFA) content was analyzed using HPLC (Agilent Technologies, USA) attached to a U.V. detector at $210 \mathrm{~nm}$. The column was an ion-exclusion Aminex HPX-87H (7.8 x $300 \mathrm{~mm}$; Bio-Rad, USA) maintained at $50^{\circ} \mathrm{C}$ with a column heater. The eluent, $0.005 \mathrm{mM}$ sulphuric acid in HPLC-grade water, was pumped through the column at a flow rate of $0.6 \mathrm{ml} \mathrm{min}^{-1}$, and data were acquired using Chem Station for LC3D software (Agilent Technologies). Quantification of the samples was carried out using calibration curves of lactic acid at concentrations of $2.5,25$ and $50 \mathrm{mM}$ and for acetic, propionic and butyric acids at concentrations of 10, 20, 40, 80 and $100 \mathrm{mM}$ [10].

\section{Statistical analysis}

Statistical analysis was performed using SPSS version 20 for Windows. Univariate analysis of variance (ANOVA) and Tukey's multivariate comparison tests were also used to determine significant differences among the bacterial populations using the different samples and used to determine a significant increase in each SCFA concentrations. The differences were considered to be significant when $\mathrm{P}<0.05$.

\section{RESULTS:}

\section{$\beta$-glucan content}

The soluble $\beta$-glucans derived from extraction of mushrooms (Schizophylum commune Fr and Auricularia auricula Judae) by water under high pressure $\left(1.5 \mathrm{lb} \mathrm{inch}^{-1}\right)$ and high temperature $\left(130{ }^{\circ} \mathrm{C}\right)$ for 30 and $90 \mathrm{~min}$. Total $\beta$-glucans of extractable solid from Schizophylum commune $\mathrm{Fr}$ and Auricularia auricula Judae were $13.43 \pm 0.02 \%$ and $19.99 \pm 0.39 \%$, respectively. However, total $\beta$-glucans from both mushrooms were lower than those from commercial yeast $\beta$-glucan. The content of oligo- $\beta$-glucan from Schizophylum commune Fr was increased from $13.43 \pm 0.02 \%$ to $16.43 \pm 0.32 \%$ meanwhile it was decreased from $19.99 \pm 0.39 \%$ to $13.68 \pm 0.08 \%$ for Auricularia auricula Judae (Table 1). This might due to laminarinase that it was used for hydrolysis of $\beta$ glucan could deliberate $\beta$-glucan that trapped in hemicellulose matrix of Schizophylum commune.

Table 1. Content of $\beta$-glucan and oligo- $\beta$-glucan in extract of Schizophylum commune Fr and Auricularia auricula Judae

\begin{tabular}{lc}
\hline Source of $\beta$-glucan and oligo- $\beta$-glucans & \% $\boldsymbol{\beta}$-glucan (dry basis) \\
\hline$\beta$-glucan from Schizophylum commune $\mathrm{Fr}$ & $13.43 \pm 0.02$ \\
Oligo- $\beta$-glucan from Schizophylum commune $\mathrm{Fr}$ & $16.43 \pm 0.32$ \\
$\beta$-glucan from Auricularia auricula Judae & $19.99 \pm 0.39$ \\
Oligo- $\beta$-glucan from Auricularia auricula Judae & $13.68 \pm 0.08$ \\
$\beta$-glucan from Saccharomyces cerevisiae (commercial) & $49.18 \pm 0.46$ \\
\hline
\end{tabular}




\section{Batch culture fermentation}

Batch culture fermentation was used to monitor the effect of $\beta$-glucan and oligo- $\beta$-glucans addition on the mixed bacterial populations. Samples were removed at 0,24 and $48 \mathrm{~h}$ for quantifying the levels of different bacterial groups by FISH technique. The results indicated that a significant increase in the levels of bifidobacteria and lactobacillus was observed with $\beta$-glucan from Auricularia auricula Judae after $24 \mathrm{~h}$ of incubation (Table 2). Generally, a decrease in bacteroides and clostridia were observed in response to both $\beta$-glucans from Auricularia auricula Judae and Schizophylum commune Fr. Oligo- $\beta$-glucan from Schizophylum commune Fr was shown decrease in the number of lactobacilli both $\beta$-glucan from Auricularia auricula Judae and $\beta$-glucan from Schizophylum commune Fr. Commercial yeast $\beta$-glucan resulted in an increase the numbers of bifidobacterial, clostridia and decreased in eubacteria, lactobacilli and bacteroides.

Table 2. Bacterial populations (log cell $\mathrm{mL}^{-1}$ ) change during fecal fermentation of $\beta$-glucan and oligo- $\beta$-glucan in batch culture

\begin{tabular}{|c|c|c|c|c|c|c|}
\hline Sample & $\begin{array}{l}\text { Time } \\
\text { (hour) }\end{array}$ & Bifidobacteria & Lactobacillus & Bacteroides & Clostridia & Eubacteria \\
\hline \multirow{5}{*}{$\begin{array}{l}\beta \text {-glucan from } \\
\text { Schizophylum } \\
\text { commune Fr }\end{array}$} & & & & & & \\
\hline & & & & & & \\
\hline & 0 & $10.34 \pm 1.24 \mathrm{ab}$ & $10.91 \pm 1.01 \mathrm{~b}$ & $11.04 \pm 0.79 b$ & $11.07 \pm 1.25 b$ & $11.49 \pm 0.89 \mathrm{a}$ \\
\hline & 24 & $10.26 \pm 1.18^{b}$ & $10.88 \pm 0.80^{\mathrm{ab}}$ & $11.28 \pm 1.03^{\mathrm{c}}$ & $11.15 \pm 1.23^{\mathrm{c}}$ & $11.51 \pm 0.98^{\mathrm{a}}$ \\
\hline & 48 & $10.00 \pm 1.09^{\mathrm{a}}$ & $10.85 \pm 0.83^{\mathrm{a}}$ & $10.81 \pm 0.72^{\mathrm{a}}$ & $10.99 \pm 1.13^{\mathrm{a}}$ & $11.50 \pm 0.95^{\mathrm{a}}$ \\
\hline \multicolumn{7}{|l|}{ Oligo- $\beta$-glucan from } \\
\hline \multirow{4}{*}{$\begin{array}{l}\text { Schizophylum } \\
\text { commune Fr }\end{array}$} & & & & & & \\
\hline & 0 & $9.96 \pm 1.01^{\mathrm{a}}$ & $10.59 \pm 0.91^{\mathrm{b}}$ & $10.95 \pm 1.14^{\mathrm{b}}$ & $11.00 \pm 1.09^{\mathrm{a}}$ & $11.18 \pm 0.73^{\mathrm{a}}$ \\
\hline & 24 & $10.01 \pm 0.91^{\mathrm{a}}$ & $10.58 \pm 0.83^{\mathrm{ab}}$ & $11.09 \pm 1.27^{\mathrm{c}}$ & $11.10 \pm 1.28^{\mathrm{b}}$ & $11.51 \pm 1.16^{\mathrm{b}}$ \\
\hline & 48 & $10.10 \pm 0.54^{\mathrm{a}}$ & $10.52 \pm 0.81^{\mathrm{a}}$ & $11.02 \pm 0.84^{\mathrm{a}}$ & $10.95 \pm 1.25^{\mathrm{a}}$ & $11.54 \pm 0.96^{\mathrm{c}}$ \\
\hline \multirow{2}{*}{\multicolumn{7}{|c|}{$\begin{array}{l}\beta \text {-glucan from } \\
\text { Auricularia auricula }\end{array}$}} \\
\hline & & & & & & \\
\hline \multirow[t]{3}{*}{ Judae } & 0 & $10.54 \pm 1.27^{\mathrm{a}}$ & $10.99 \pm 0.96^{\mathrm{a}}$ & $10.94 \pm 1.07^{\mathrm{b}}$ & $11.16 \pm 1.16^{\mathrm{c}}$ & $11.27 \pm 0.93^{\mathrm{a}}$ \\
\hline & 24 & $10.59 \pm 1.30^{\mathrm{b}}$ & $11.00 \pm 0.97^{\mathrm{a}}$ & $10.74 \pm 0.63^{\mathrm{a}}$ & $11.04 \pm 1.07^{\mathrm{b}}$ & $11.38 \pm 0.88^{b}$ \\
\hline & 48 & $10.11 \pm 1.24^{\mathrm{b}}$ & $11.15 \pm 0.91^{\mathrm{b}}$ & $10.71 \pm 0.55^{\mathrm{a}}$ & $10.80 \pm 0.85^{\mathrm{a}}$ & $11.30 \pm 0.89^{\mathrm{a}}$ \\
\hline \multicolumn{7}{|l|}{ Oligo- $\beta$-glucan from } \\
\hline \multirow{4}{*}{$\begin{array}{l}\text { Auricularia auricula } \\
\text { Judae }\end{array}$} & & & & & & $11.34 \pm 1.00^{\mathrm{a}}$ \\
\hline & 0 & $9.64 \pm 0.79^{c}$ & $10.78 \pm 0.55^{\mathrm{a}}$ & $11.00 \pm 1.07^{\mathrm{a}}$ & $11.01 \pm 1.04^{\mathrm{a}}$ & \\
\hline & 24 & $9.90 \pm 0.92^{\mathrm{a}}$ & $10.88 \pm 0.80^{\mathrm{b}}$ & $11.25 \pm 1.02^{\mathrm{b}}$ & $11.28 \pm 1.30^{\mathrm{b}}$ & $11.57 \pm 0.96^{\mathrm{b}}$ \\
\hline & 48 & $10.37 \pm 0.88^{\mathrm{b}}$ & $10.81 \pm 0.73^{\mathrm{a}}$ & $11.43 \pm 1.25^{\mathrm{c}}$ & $11.22 \pm 1.25^{\mathrm{c}}$ & $11.57 \pm 1.23^{\mathrm{b}}$ \\
\hline \multirow{4}{*}{$\begin{array}{l}\text { Commercial yeast } \\
\beta \text {-glucan }\end{array}$} & & & & & & $11.39 \pm 1.00^{\mathrm{b}}$ \\
\hline & 0 & $10.72 \pm 1.36^{\mathrm{a}}$ & $11.15 \pm 0.97^{\mathrm{b}}$ & $10.93 \pm 0.83^{b}$ & $11.18 \pm 1.28^{\mathrm{a}}$ & \\
\hline & 24 & $10.70 \pm 1.22^{\mathrm{b}}$ & $11.23 \pm 1.00^{c}$ & $10.96 \pm 0.97^{\mathrm{b}}$ & $11.35 \pm 1.30^{\mathrm{b}}$ & $11.49 \pm 1.00^{\mathrm{c}}$ \\
\hline & 48 & $9.96 \pm 1.01^{\mathrm{b}}$ & $11.07 \pm 0.93^{\mathrm{a}}$ & $10.75 \pm 0.70^{\mathrm{a}}$ & $11.34 \pm 1.29^{\mathrm{b}}$ & $11.27 \pm 0.82^{\mathrm{a}}$ \\
\hline
\end{tabular}

* Different letters in a column means are significantly different $(\mathrm{p}<0.05)$ 


\section{Prebiotic index (PI)}

The Prebiotic Index (PI) represents a comparative relationship between the growth of "beneficial" bacteria, such as bifidobacteria and lactobacilli, and that of the "undesirable" bacteria, such as clostridia and bacteroides, in relation to the changes of the total number of bacteria represented by eubacteria (Figure 1). PI values at $48 \mathrm{~h}$ of incubation were higher than those at $24 \mathrm{~h}$ in all samples. The $\beta$-glucan from Auricularia auricula Judae fraction produced the greatest PI values, followed by commercial yeast $\beta$-glucan and $\beta$-glucan from Schizophylum commune Fr, respectively. Meanwhile oligo- $\beta$-glucan is not a candidate prebiotic. The PI at $48 \mathrm{~h}$ of $\beta$-glucan and oligo- $\beta$-glucan from Schizophylum commune Fr were 0.01 and -0.01, respectively. $\beta$-glucan and oligo- $\beta$-glucan from Auricularia auricula Judae were 0.11 and -0.07, respectively. Whereas PI of $\beta$-glucan from commercial yeast (Saccharomyces cerevisiae) was 0.03 .

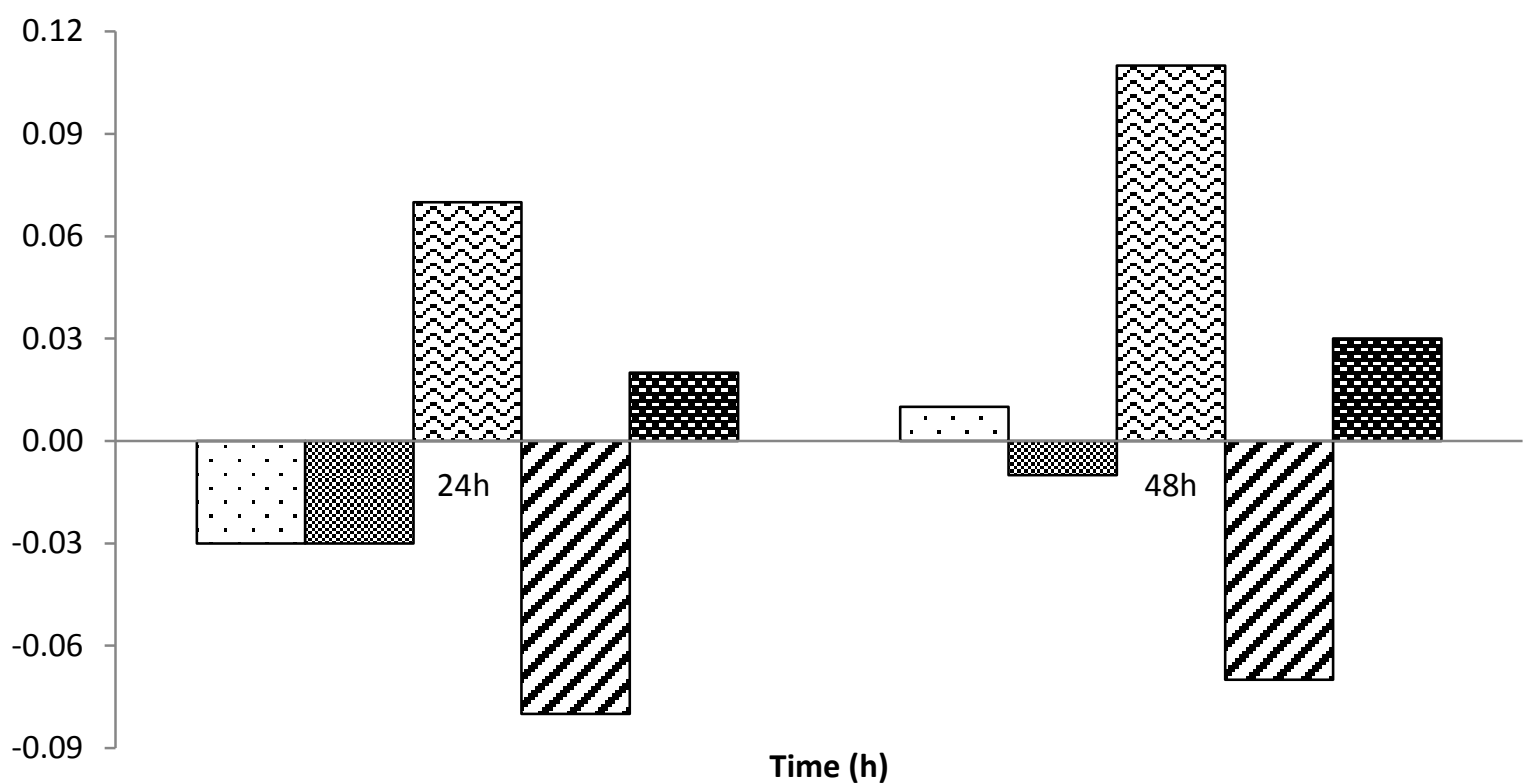

$\square$ Schizophylum commune Fr $\beta$-glucan

Schizophylum commune Fr oligo- $\beta$-glucan $\square$ Auricularia auricula Judae $\beta$-glucan $\square$ Auricularia auricula Judae oligo- $\beta$-glucan

Yeast $\beta$-glucan commercial

Figure 1. Prebiotic Index (PI) scores from batch cultures of $\beta$-glucan and oligo- $\beta$-glucan from mushrooms

\section{Short chain fatty acid production}

Acetate was the most prevalent SCFA found in all samples followed by propionate, butyrate and lactate, respectively (Table 3 ). Acetate production from oligo- $\beta$-glucan was also higher, both in terms of total amounts and proportions of total SCFA produced, in comparison with $\beta$-glucan. Lactate was not produced from any sample tested otherwise it was rapidly converted into others SCFA. The cross-feeding of other bacteria by lactate which is the likely cause of its decline in concentration by $48 \mathrm{~h}$. However, fermentation of $\beta$-glucan and oligo- $\beta$-glucan resulted in higher total amounts and proportions of acetate; ranging from 54.64-68.01\% with $\beta$-glucan, to 63.30- 
$79.04 \%$ with oligo- $\beta$-glucan, at $48 \mathrm{~h}$. The profiles of each SCFA were difference however in general ratio of acetate: propionate: butyrate for all $\beta$-glucan and oligo- $\beta$-glucan were in range of 4: 2: 1 (Table 4).

Table 3. Concentration $(\mathrm{mM})$ of short chain fatty acid produced by fecal fermentation of $\beta$ glucan and oligo- $\beta$-glucan in batch culture

\begin{tabular}{|c|c|c|c|c|c|c|c|}
\hline Treatment & $\begin{array}{l}\text { Time } \\
\text { (hour) }\end{array}$ & Total SCFA & Lactate & Acetate & Propionate & Butyrate & $\begin{array}{l}\text { Butyrate/ } \\
\text { Acetate }\end{array}$ \\
\hline \multicolumn{8}{|l|}{$\beta$-glucan from } \\
\hline \multicolumn{8}{|l|}{ Schizophylum } \\
\hline \multirow{3}{*}{ commune Fr } & 0 & $30.56 \pm 2.48^{\mathrm{a}}$ & $2.91 \pm 0.09^{b}$ & $7.32 \pm 1.31^{\mathrm{a}}$ & $20.33 \pm 1.43^{\mathrm{b}}$ & $0.00 \pm 0.00^{\mathrm{b}}$ & $0.00 \pm 0.00^{\mathrm{a}}$ \\
\hline & 24 & $38.59 \pm 4.37^{\mathrm{a}}$ & $0.00 \pm 0.00^{\mathrm{a}}$ & $22.51 \pm 1.88^{b}$ & $10.37 \pm 1.78^{\mathrm{a}}$ & $5.71 \pm 0.84^{\mathrm{a}}$ & $0.25 \pm 0.02^{\mathrm{b}}$ \\
\hline & 48 & $61.07 \pm 9.24^{\mathrm{b}}$ & $0.00 \pm 0.00^{\mathrm{a}}$ & $33.37 \pm 4.91^{\mathrm{c}}$ & $17.49 \pm 2.80^{\mathrm{b}}$ & $10.21 \pm 1.54^{\mathrm{b}}$ & $0.31 \pm 0.00^{\mathrm{c}}$ \\
\hline \multicolumn{8}{|l|}{$\begin{array}{l}\text { Oligo- } \beta \text {-glucan } \\
\text { from }\end{array}$} \\
\hline \multicolumn{8}{|l|}{ Schizophylum } \\
\hline \multirow[t]{3}{*}{ commune Fr } & 0 & $41.94 \pm 3.76^{\mathrm{a}}$ & $3.42 \pm 0.13^{b}$ & $15.53 \pm 1.72^{\mathrm{a}}$ & $22.99 \pm 2.18^{\mathrm{b}}$ & $0.00 \pm 0.00^{\mathrm{b}}$ & $0.00 \pm 0.00^{\mathrm{a}}$ \\
\hline & 24 & $65.82 \pm 3.64^{b}$ & $0.00 \pm 0.00^{\mathrm{a}}$ & $41.42 \pm 1.22^{\mathrm{b}}$ & $14.56 \pm 1.55^{\mathrm{a}}$ & $9.83 \pm 0.90^{\mathrm{a}}$ & $0.24 \pm 0.01^{b}$ \\
\hline & 48 & $79.47 \pm 0.34^{\mathrm{c}}$ & $0.00 \pm 0.00^{\mathrm{a}}$ & $50.03 \pm 0.32^{\mathrm{c}}$ & $17.36 \pm 0.23^{\mathrm{a}}$ & $11.81 \pm 0.31^{\mathrm{a}}$ & $0.24 \pm 0.01^{b}$ \\
\hline$\beta$-glucan from & 0 & & & & & & \\
\hline \multicolumn{8}{|l|}{ Auricularia } \\
\hline \multirow[t]{3}{*}{ auricula Judae } & & $3.64 \pm 0.86^{\mathrm{a}}$ & $1.20 \pm 0.33^{\mathrm{b}}$ & $2.44 \pm 0.53^{\mathrm{a}}$ & $0.00 \pm 0.00^{\mathrm{a}}$ & $0.00 \pm 0.00^{\mathrm{a}}$ & $0.00 \pm 0.00^{\mathrm{a}}$ \\
\hline & 24 & $27.11 \pm 0.81^{b}$ & $0.00 \pm 0.00^{\mathrm{a}}$ & $19.05 \pm 0.55^{\mathrm{b}}$ & $4.69 \pm 0.04^{\mathrm{b}}$ & $3.36 \pm 0.24^{\mathrm{b}}$ & $0.18 \pm 0.01^{b}$ \\
\hline & 48 & $38.42 \pm 1.53^{\mathrm{c}}$ & $0.00 \pm 0.00^{\mathrm{a}}$ & $26.13 \pm 0.97^{\mathrm{c}}$ & $7.00 \pm 0.33^{\mathrm{c}}$ & $5.29 \pm 0.27^{\mathrm{c}}$ & $0.20 \pm 0.00^{\mathrm{c}}$ \\
\hline \multicolumn{8}{|l|}{$\begin{array}{l}\text { Oligo- } \beta \text {-glucan } \\
\text { from }\end{array}$} \\
\hline \multicolumn{8}{|l|}{ Auricularia } \\
\hline \multirow[t]{3}{*}{ auricula Judae } & 0 & $61.80 \pm 0.26^{\mathrm{a}}$ & $1.05 \pm 0.10^{\mathrm{b}}$ & $57.12 \pm 0.13^{\mathrm{b}}$ & $3.63 \pm 0.37^{\mathrm{a}}$ & $0.00 \pm 0.00^{\mathrm{a}}$ & $0.00 \pm 0.00^{\mathrm{a}}$ \\
\hline & 24 & $65.62 \pm 0.43^{b}$ & $0.00 \pm 0.00^{\mathrm{a}}$ & $56.25 \pm 0.33^{\mathrm{b}}$ & $5.19 \pm 0.26^{\mathrm{b}}$ & $4.18 \pm 0.43^{\mathrm{b}}$ & $0.07 \pm 0.01^{b}$ \\
\hline & 48 & $45.46 \pm 0.44^{\mathrm{c}}$ & $0.00 \pm 0.00^{\mathrm{a}}$ & $35.93 \pm 0.60^{\mathrm{a}}$ & $5.14 \pm 0.05^{\mathrm{b}}$ & $4.39 \pm 0.20^{\mathrm{b}}$ & $0.12 \pm 0.01^{\mathrm{c}}$ \\
\hline \multirow[t]{3}{*}{$\begin{array}{l}\text { Yeast } \beta \text {-glucan } \\
\text { commercial }\end{array}$} & 0 & $683+0.78^{\mathrm{a}}$ & $106+0.12^{\mathrm{b}}$ & $299+036^{\mathrm{a}}$ & $279+031^{\mathrm{a}}$ & $000+0.00^{\mathrm{a}}$ & $000+000^{\mathrm{a}}$ \\
\hline & 24 & $66.60 \pm 2.88^{b}$ & $0.00 \pm 0.00^{\mathrm{a}}$ & $41.06 \pm 1.72^{\mathrm{b}}$ & $17.37 \pm 0.88^{\mathrm{b}}$ & $8.18 \pm 0.31^{\mathrm{b}}$ & $0.20 \pm 0.00^{\mathrm{b}}$ \\
\hline & 48 & $64.97 \pm 0.20^{\mathrm{b}}$ & $0.00 \pm 0.00^{\mathrm{a}}$ & $39.26 \pm 0.03^{\mathrm{b}}$ & $16.69 \pm 0.12^{\mathrm{b}}$ & $9.02 \pm 0.10^{\mathrm{b}}$ & $0.23 \pm 0.00^{\mathrm{c}}$ \\
\hline
\end{tabular}

$*$ Different letters in a column means are significantly different $(\mathrm{p}<0.05)$

Table 4. Acetate, propionate and butyrate profile at $48 \mathrm{~h}$ fecal fermentation of $\beta$-glucan and oligo- $\beta$-glucan in batch culture

\begin{tabular}{llcc}
\hline & \multicolumn{3}{c}{ \% of total SCFA } \\
\cline { 2 - 4 } Treatment & Acetate & Propionate & Butyrate \\
\hline$\beta$-glucan from Schizophylum commune $\mathrm{Fr}$ & 54.64 & 28.64 & 16.71 \\
Oligo- $\beta$-glucan from Schizophylum commune $\mathrm{Fr}$ & 63.30 & 21.84 & 14.86 \\
$\beta$-glucan from Auricularia auricula Judae & 68.01 & 18.21 & 13.78 \\
Oligo- $\beta$-glucan from Auricularia auricula Judae & 79.04 & 11.31 & 9.65 \\
Commercial yeast $\beta$-glucan & 60.43 & 25.69 & 13.88 \\
\hline
\end{tabular}




\section{DISCUSSION:}

The present study has demonstrated the prebiotic potential of $\beta$-glucan from Schizophylum commune Fr and Auricularia auricula Judae. Recently, it has been demonstrated that polysaccharides obtained from mushrooms such as Pleurotus sp., Lentinus edodes, Tremella fuciformis and Agaricus bisporus have prebiotic activity. The active component is believed to be the long chain $\beta$-glucans, including homo-glucans and hetero-glucans with $\beta(1 \rightarrow 3), \beta(1 \rightarrow 4)$, and $\beta(1 \rightarrow 6)$ glucosidic linkages [8]. Mushrooms extract of $P$. ostreatus and $P$. eryngii were able to stimulate the growth of probiotics such as Lactobacillus ssp. (4 strains: Lac A-D), Bifidobacterium ssp.(3 strains: Bifi A-C) and Enterococcus faecium (2 strains: Ent A and B) to some extent. Maximum growth rate, maximum biomass concentration and final acid production were observed in the study. It was found that extract from $P$. eryngi support the growth of Lactobacillus strains better than $P$. ostreatus. Lactobacillus B and C showed the highest production of short chain fatty acid (SCFA), while Bifidobacteria A showed the lowest amount of SCFA when supplemented with both extracts.

Prebiotics are non-digested food ingredients that beneficially affects the host by selectively stimulating the growth and/or activity of one or a limited number of bacteria in the colon", that can improve the host health. One of the strongest health benefits proposed for prebiotics is the barrier function against invading gastrointestinal pathogens, such as campylobacters, salmonellas and Escherichia coli [1]. The usual target species for such a dietary intervention are bifidobacteria and lactobacillus. These organisms have been reported to have health-promoting properties. Many studies have now confirmed that prebiotics are a valid approach to the dietary manipulation of the colonic microflora [2]. Therefore, attempts to identify and develop novel, enhanced prebiotics as functional foods that are able to modulate the composition of human colonic microflora are currently of great interest.

Fermentation studies of beta-glucans can indeed shed light on the possible use of betaglucans as a novel source of long chain prebiotics. For example, the fermentability of oat betaglucan to produce SCFAs points to their potential application as a prebiotic in promoting human health [11]. In the late 1990s and early 2000s, beta-glucans have been reported as a potential prebiotic, selectively promoting the growth of beneficial intestinal microorganisms such as lactobacilli and bifidobacteria according to some in vitro studies and animal experiments [8].

In this study, the majority of the bifidobacteria and lactobacillus grew better on the $\beta$-glucan from Auricularia auricula Judae compared to the other bacteria. On the other hand, all of the Bacteroides and Clostridium species showed very low growth on the $\beta$-glucan from Auricularia auricula Judae and $\beta$-glucan from Schizophylum commune Fr substrates. This clearly indicates that the $\beta$-glucan may allow a prebiotic effect by preferentially enhancing the growth of bifidobacteria and reducing the growth of bacteroides and clostridia. The presence of $\beta$-glucan from Auricularia auricula Judae induced the growth of both bifidobacteria and lactobacilli, while only bifidobacteria growth was enhanced with oligo- $\beta$-glucan from Auricularia auricula Judae.

The PI values at 24 and $48 \mathrm{~h}$ of $\beta$-glucan from mushrooms were higher than $\beta$-glucan from yeast and oligo- $\beta$-glucan. This is probably due to a different composition and also variation in the bacterial composition of the faecal material used; oligo- $\beta$-glucan being utilized faster than $\beta$ - 
glucan. This study demonstrated that $\beta$-glucan from mushrooms (Schizophylum commune Fr and Auricularia auricula Judae) are candidate prebiotics.

Short chain fatty acids, acetate was the most prevalent SCFA found in all samples followed by propionate, butyrate and lactate, respectively. A previous in vitro study using rat caecal inocula to ferment (predigested) oat bran resulted in a SCFA ratio of 69: 19: 12 for acetate: propionate: butyrate. This was considered a high proportion of propionate in comparison with the SCFA profile generated by other substrates (NB acetate is the dominant SCFA in most fermentation studies of this nature). This may have resulted from the high purity $(>96 \%)$ of the $\beta$-glucan being fermented, compared with the more crude forms of $\beta$-glucan (e.g. oat bran). Another study investigating the fermentation of relatively pure arabinoxylans (>94\%) demonstrated an average SCFA ratio of 63: 12: 25, which was deemed an example of a butyrateenriched SCFA profile. If SCFA, and in particular propionate, are implicated in the hypocholesterolaemic effect of $\beta$-glucan it is necessary to determine the quantity and ratio of different SCFAs produced from the fermentation of relatively pure $\beta$-glucan fractions (unlike previous studies that used oats, or oat bran concentrate). Furthermore, it is important to correlate the specific groups of the microbial communities associated with $\beta$-glucan fermentation and the consequential SCFA profiles [4].

Conclusion: The study confirmed that $\beta$-glucan from Schizophylum commune Fr and Auricularia auricula Judae are candidate prebiotics.

Competing Interests: The authors declare that they have no conflicts of interest.

Author's Contributions: All authors contributed to these studies.

Acknowledgments: I would like to thanks a grant agency Agricultural Research Development Agency (ARDA, Thailand) for financial support of this project.

\section{REFERENCES:}

1. Gibson, G.R. and Roberfroid, M.B. 1995. Dietary modulation of the human colonic microbiota: introducing the concept of prebiotics. J Nutr. 125: 1401-1412.

2. Rycroft, C.E., Jones, M.R., Gibson, G.R. and Rastall, R.A. 2001. A comparative in vitro evaluation of the fermentation properties of prebiotic oligosaccharides. J Appl Microbial. 91: 878-887.

3. Tuohy, K.M., Kolida, S., Lustenberger, A.M. and Gibson, G.R. 2001. The prebiotic effects of biscuits containing partially hydrolysed guar gum and fructo-oligosaccharidesa human volunteer study. Brit J Nutr. 86: 341-348.

4. Hughes, S.A., Shewry, P.R., Gibson, G.R., McCleary, B.V. and Rastall, R.A. In vitro fermentation of oat and barley derived $\beta$-glucans by human faecal microbiota. FEMS Microbiol Ecol. 2008, 64: 482-493.

5. Zhao, J. and Cheung, P.C.K. Fermentation of $\beta$-glucans derived from different sources by bifidobacteria: Evaluation of their bifidogenic effect. J Agr Food Chem. 2011. 59: 5986-5992. 
6. Volman, J. J., Ramakers, J. D. and Plat, J. 2008. Dietary modulation of immune function by $\beta$-glucans. Physiol Behav. 94: 276-284.

7. Zhao, J. and Cheung, P.C.K. 2013. Comparative Proteome Analysis of Bifidobacterium longum subsp. infantis Grown on $\beta$-Glucans from Different Sources and a Model for Their Utilization. J Agr Food Chem. 61: 4360-4370.

8. Lam, K.L., Cheung, P.C.K. 2013. Non-digestible long chain $\beta$-glucans as novel prebiotics. Bioactive Carbohydrates and Dietary Fibre. 2: 45-64.

9. Palframan, R., Gibson, G. R. and Rastall, R. A. 2002. Effect of $\mathrm{pH}$ and dose on the growth of gut bacteria on prebiotic carbohydrates in vitro. Anaerobe. 8: 287-292.

10. Olano-Martin, E., Mountzouris, K.C., Gibson, G.R. and Rastall, R.A. 2000. In vitro fermentability of dextran, oligodextran and maltodextrin by human gut bacteria. Brit $\mathbf{J}$ Nutr. 83: 247-255.

11. Lin, B., Gong, J., Wang, Q., Cui, S., Yu, H. and Huang, B. 2011. In vitro assessment of the effects of dietary fibers on microbial fermentation and communities from large intestinal digesta of pigs. Food Hydrocolloids, 25: 180-188. 